\title{
Proteomic Analysis of the Peri-Infarct Area after Human Umbilical Cord Mesenchymal Stem Cell Transplantation in Experimental Stroke
}

\author{
Dongsheng He ${ }^{1-3,5,6 \#}$, Zhuo Zhang ${ }^{4 \#}$, Jiamin Lao ${ }^{1-3,5,6 \#}$, Hailan Meng ${ }^{1-3,5,6}$, Lijuan Han ${ }^{1-3,5,6}$, Fan \\ Chen ${ }^{1-3,5,6}$, Dan Ye $\mathrm{Y}^{1-3,5,6}$, He Zhang ${ }^{1-3,5,6}$, Yun $\mathrm{Xu}^{1-3,5,6^{*}}$
}

${ }^{1}$ Department of Neurology, Affiliated Drum Tower Hospital, and ${ }^{2}$ Jiangsu Key Laboratory for Molecular Medicine, Nanjing University Medical School, Nanjing 210008, China.

${ }^{3}$ The State Key Laboratory of Pharmaceutical Biotechnology, Nanjing University, Nanjing 210008, China

${ }^{4}$ Department of Gastroenterology, Children's Hospital of Nanjing, Nanjing Medical University, Nanjing 210008, China

${ }^{5}$ Jiangsu Province Stroke Center for Diagnosis and Therapy, Nanjing 210008, China

${ }^{6}$ Nanjing Neuropsychiatry Clinic Medical Center, Nanjing 210008, China

${ }^{\#}$ These authors contributed equally to this work

[Received November 15, 2015; Revised December 15, 2015; Accepted January 21, 2016]

\begin{abstract}
Among various therapeutic approaches for stroke, treatment with human umbilical cord mesenchymal stem cells (hUC-MSCs) has acquired some promising results. However, the underlying mechanisms remain unclear. We analyzed the protein expression spectrum of the cortical peri-infarction region after ischemic stroke followed by treatment with hUC-MSCs, and found 16 proteins expressed differentially between groups treated with or without hUC-MSCs. These proteins were further determined by Gene Ontology term analysis and network with CD200-CD200R1, CCL21-CXCR3 and transcription factors. Three of them: Abca13, Grb2 and Ptgds were verified by qPCR and ELISA. We found the protein level of Abca13 and the mRNA level of Grb2 consistent with results from the proteomic analysis. Finally, the function of these proteins was described and the potential proteins that deserve to be further studied was also highlighted. Our data may provide possible underlying mechanisms for the treatment of stroke using hUC-MSCs.
\end{abstract}

Key words: stroke, hUC-MSCs, proteomics, iTRAQ, transplantation, human

Stroke is a leading cause of disability in China as well as in Western countries. Unfortunately, although much attention has been given to it, there is still a lack of effective treatments. Cell-based therapy for stroke, frequently studied in recent years, has shown promising results. For example, ischemic stroke treated with mesenchymal stem cells (MSCs) could improve functional outcome in animal studies and clinical settings
[1-7]. In animal studies, the neurological deficit induced by ischemic stroke is significantly improved while infarct volume is significantly attenuated after transplantation of MSCs. Similarly, a clinical trial conducted by Lee and colleagues showed that neurological deficits of stroke patients transplanted with autologous MSCs were improved without cell-related adverse events during the short-term and long-term follow up periods. Although the

*Correspondence should be addressed to: Yun Xu MD, PhD, Department of Neurology, Affiliated Drum Tower Hospital of Nanjing University Medical School, Nanjing 210008, China. Email: xuyun20042001@aliyun.com

Copyright: ( 2016 He D. et al. This is an open-access article distributed under the terms of the Creative Commons Attribution License, which permits unrestricted use, distribution, and reproduction in any medium, provided the original author and source are credited. 
findings are encouraged, the molecular mechanisms remain obscure. Recent studies have documented that MSCs have the capacity to pass through the blood-brain barrier and migrate throughout the forebrain and cerebellum, wherein the MSCs may differentiate into neuronal-like cells. The functional property of MSCsderived newborn neurons and the network between them remain to be unverified. Therefore, a hypothesis that MSCs may exert their function by producing trophic factors such as brain-derived neurotrophic factor (BDNF), vascular endothelial growth factor (VEGF), hepatocyte growth factor (HGF), nerve growth factor (NGF), basic fibroblast growth factor (bFGF, FGF-2), insulin growth factor-1 (IGF-1), and so on has been generated [8-10].

Human umbilical cord mesenchymal stem cells (hUC-MSCs) are MSCs derived from the human umbilical cord, which can be used without raising any ethnical concerns because this extra-embryonic tissue is often discarded. hUC-MSCs express specific surface antigens; positive for CD105, CD73 and CD90, and negative for CD45, CD34, CD14 or CD11b, CD79a or CD19, and HLA-DR surface molecules. Besides having multipotent differentiation potential, hUC-MSCs share a close ontogenetic relationship to embryonic stem cells (ESCs) and proliferate faster than adult MSC [11]. Our previous study [12] showed that hUC-MSCs intravenously injected into ischemic animals could significantly improve neurological function and reduce infarct volume within 30 min after middle cerebral artery occlusions (MCAO), along with reduced expression of pro-inflammatory cytokines, including IL-1, TNF- $\alpha$, IL23 and IL-17, and increased expression of the antiinflammatory cytokine such as IL-10. Interestingly, these effects could be partially inhibited by the TGF- $\beta$ neutralizing antibody.

In the present study, iTRAQ (isobaric tags for relative and absolute quantification) was used to analyze the difference of protein expression between the stroke and sham groups, with or without intravenous injection of hUC-MSCs.

\section{MATERIAL AND METHODS}

\section{Animals and MCAO model}

Six to seven week old male C57BL/6 mice (weighing between 25 and $30 \mathrm{~g}$ ) were provided by the Drum Tower Hospital Animal Center and used according to protocols approved by the Committee of Experimental Animal Administration of Nanjing University, China.

Mice were randomly allocated to groups as described below. The randomization procedure was performed by the RAND function in Office Excel 2010. We first assigned a random number (from 1-102) to each mouse.
Next, the mice were sorted by the random numbers (in ascending order). They were then allocated to the 34 groups according to their locations in the ranked random numbers series. The number of mice in each experimental group is listed in Table 1.

Table1. The number of mice in each experiment group.

\begin{tabular}{|c|c|c|}
\hline Experiment & Group & No. of mice \\
\hline \multirow[t]{4}{*}{ Proteomics } & sham & $1-3$ \\
\hline & MCAO 24h & $4-6$ \\
\hline & MCAO 48h & $7-9$ \\
\hline & MCAO 48h + hUC-MSCs & $10-12$ \\
\hline \multirow[t]{18}{*}{ QPCR } & Sham $6 \mathrm{~h}$ & $13-17$ \\
\hline & MCAO 6h & $18-22$ \\
\hline & MCAO + hUC-MSCs $6 \mathrm{~h}$ & $23-27$ \\
\hline & Sham $12 \mathrm{~h}$ & $28-32$ \\
\hline & MCAO $12 \mathrm{~h}$ & $33-37$ \\
\hline & MCAO + hUC-MSCs $12 \mathrm{~h}$ & $38-42$ \\
\hline & Sham $24 \mathrm{~h}$ & $43-47$ \\
\hline & MCAO 24h & $48-52$ \\
\hline & $\mathrm{MCAO}+\mathrm{hUC}-\mathrm{MSCs} 24 \mathrm{~h}$ & $53-57$ \\
\hline & Sham $48 \mathrm{~h}$ & $58-62$ \\
\hline & MCAO 48h & $63-67$ \\
\hline & MCAO + hUC-MSCs $48 \mathrm{~h}$ & $68-72$ \\
\hline & Sham $72 \mathrm{~h}$ & $73-77$ \\
\hline & MCAO 72h & $78-82$ \\
\hline & MCAO + hUC-MSCs 72h & $83-87$ \\
\hline & Sham 1W & $88-92$ \\
\hline & MCAO 1W & $93-97$ \\
\hline & MCAO + hUC-MSCs $1 \mathrm{~W}$ & $98-102$ \\
\hline \multirow[t]{12}{*}{ ELISA } & Sham $24 \mathrm{~h}$ & $43-47$ \\
\hline & MCAO $24 \mathrm{~h}$ & $48-52$ \\
\hline & MCAO + hUC-MSCs $24 \mathrm{~h}$ & $53-57$ \\
\hline & Sham $48 \mathrm{~h}$ & $58-62$ \\
\hline & MCAO 48h & $63-67$ \\
\hline & MCAO + hUC-MSCs 48h & $68-72$ \\
\hline & Sham $72 \mathrm{~h}$ & $73-77$ \\
\hline & MCAO 72h & $78-82$ \\
\hline & $\mathrm{MCAO}+\mathrm{hUC}-\mathrm{MSCs} 72 \mathrm{~h}$ & $83-87$ \\
\hline & Sham 1W & $88-92$ \\
\hline & MCAO $1 \mathrm{~W}$ & $93-97$ \\
\hline & MCAO + hUC-MSCs 1W & 98-102 \\
\hline
\end{tabular}

Focal cerebral ischemia was induced by MCAO as previously described [13]. Briefly, after mice were anaesthetized by intraperitoneal injection of sodium pentobarbital $(1 \%)$ at a dose of $45 \mathrm{mg} / \mathrm{kg}$, a midline cervical incision was made under a dissecting microscope and then the right common carotid artery and external carotid artery were isolated. Next, a poly-L-lysine coated nylon monofilament thread was inserted through the 
external carotid artery and advanced into the internal carotid artery to occlude the origin of the middle cerebral artery (approximately $12 \mathrm{~mm}$ ). During the procedure, a homeothermic blanket and water pads were used to maintain body and head temperatures at $37 \pm 0.5^{\circ} \mathrm{C}$. After $90 \mathrm{~min}$ of occlusion, the filament was withdrawn for reperfusion. Sham-operated mice were subjected to the same procedure without MCAO.

\section{Preparation and injection of hUC-MSCs}

hUC-MSCs from passages 2 to 3 (Shenzhen Beike Stem Cell Engineering Institute) were used for this study [14]. $4 \times 10^{6}$ hUC-MSCs was injected via the tail vein within 30min after ischemia/reperfusion (I/R). While the same volume of NS was used as control.

\section{Sample preparation}

Mice were sacrificed at the indicated time points under deep anesthesia using halothane (0.3-0.5 ml per mouse). The brains were removed quickly and the cortical periinfarct areas were harvested and stored at $-80^{\circ} \mathrm{C}$ for further analysis. At indicated time points, the acquired cortex was divided into 2 parts for quantitative real-time PCR (qPCR) and ELISA respectively as shown in Table 1.

\section{iTRAQ}

A detailed protocol for proteomics analysis was followed as previously described [15]. Briefly, iTRAQ labeling (Applied Biosystems) coupled with online twodimensional Nano LC/MS/MS system (2D-nanoLCMS/MS) (Agilent, Waldbronn, Germany) was used; protein $(100 \mu \mathrm{g})$ from each sample was denatured, alkylated, and digested before being labeled with iTRAQ reagents and mixed. After being cleaned, desalted, and vacuum-dried, the mixed sample was analyzed using the On-line two-dimensional Nano LC/MS/MS on a NanoHPLC system coupled to a hybrid Q-TOF mass spectrometer (QSTAR XL, Applied Biosystems) equipped with a Nano-ESI source (Applied Biosystems) and a Nano-ESI needle (Picotip, FS360-50- 20; New Objective Inc., Woburn, MA). The ProteinPilot ${ }^{\mathrm{TM}}$ Software 3.0 (revision 114732) was used to analyze the differential expressed protein. A ratio more than 1.5-fold or less than 0.66-fold was deemed significant. Using the Software DAVID (http://david.abcc.ncifcrf.gov/) coupled with wego (http://wego.genomics.org.cn/cgi-bin/wego/ index.pl), the GO term analysis was completed and the differential expressed protein of each group was determined. STRING (V9.1) software was then used to evaluate the network with crosstalk key molecules and with transcription factors.

\section{qPCR}

Real-time PCR was performed as described previously [14]. Trizol reagent (Takara, life technologies, USA) was used to extract total RNA that were then reversetranscribed into cDNA using a Prime Script RT reagent kit (Takara, Clontech,USA) for quantitative PCR (Takara, Clontech, USA) in the presence of a fluorescent dye (SYBR GreenI; Takara, Clontech, USA; $\mathrm{n}=5$ per group). The relative level of mRNA was calculated and presented after normalization to glyceraldehyde-3-phosphate dehydrogenase ribosomal RNA.

The primer sequences used (Invitrogen, USA) are as follows:

Abca13 primers:

Forward: 5-'CCTGCCCTATGTGGTCCTGT-3'

Reverse: 5'-TTCCCTTCCTCCTGTCCTTCC-3'

Grb2 primers:

Forward: 5'-AGAATGGAAGCCATCGCCAA3'

Reverse: 5'-CTGCACATCATTTCCAAACGGA3'

Ptgds primers:

Forward: 5'-CAGTGGTGGAGGCCAACTAT-3'

Reverse: 5'-CCAGCCCTCTGACTGACTTC-3'

\section{ELISA}

The protein level of Abca13, Grb2 and Ptgds in all groups $(\mathrm{n}=5$ per group) were detected using mouse cytokine ELISA kits (R\&D Systems, Minneapolis, MN, USA) as described by the manufacture [14]. A microplate reader (Bio-Rad Laboratories, Hercules, CA) was used to read the optical density at $450 \mathrm{~nm}$.

\section{Statistics}

Data were expressed as mean $\pm \mathrm{SD}$. The comparisons among groups were determined by one-way analysis of variance (ANOVA) followed by Bonferroni's post hoc test. All data analyses were conducted with the SPSS 17.0 software package (SPSS, Inc., Chicago, IL, USA). A value of $p<0.05$ was considered to be statistically significant.

\section{RESULTS}

\section{Alterations in protein expression}

The cortical peri-infarct areas were harvested at $24 \mathrm{~h}$ and $48 \mathrm{~h}$ after ischemic stroke with or without treatment of hUC-MSCs. Changes in protein expression were compared between groups. 
Table 2. Differentially expressed proteins in each group

\begin{tabular}{lllll}
\hline & mcao-24h/sham & mcao-48h/sham & mcao-48h/mcao-24h & mcao-48h + hUC-MSCs/mcao-48h \\
\hline $\begin{array}{l}\text { Up-regulated } \\
\text { protein }\end{array}$ & Abca13,Ptgds, & Abca13 & $\begin{array}{l}\text { Acot11,Acat2,Grb2,Scp2, } \\
\text { Ppp2r5d,Rps12-ps11 }\end{array}$ & Rrbp1, Anxa6, Slk \\
& Hbb-b1 & & & \\
$\begin{array}{l}\text { Down- } \\
\text { regulated } \\
\text { protein }\end{array}$ & $\begin{array}{l}\text { Acot11,Acat2, } \\
\text { Grb2,Scp2, }\end{array}$ & $\begin{array}{l}\text { Acot11,Vps13d, } \\
\text { Anp32b }\end{array}$ & Vps13d,Ptgds, Hbb-b1 & Nup205, Psmd6 \\
\hline
\end{tabular}

Compared to sham group, there were 3 up-regulated and 6 down-regulated proteins at $24 \mathrm{~h}$ after cerebral ischemia; there were 1 up-regulated and 3 down-regulated proteins at $48 \mathrm{~h}$ after cerebral ischemia. Further analysis showed that there were 6 up-regulated and 3 downregulated proteins at $48 \mathrm{~h}$ after cerebral ischemia compared with the $24 \mathrm{~h}$ after cerebral ischemia group. There were also 3 up-regulated and 2 down-regulated proteins in the groups treated with hUC-MSCs compared with the $48 \mathrm{~h}$ after cerebral ischemia group.

We found that Acat2, Grb2, and Scp2 were downregulated in the mcao-24h group compared with the sham group and up-regulated in the mcao-48h group compared with the mcao-24h group. On the other hand, Ptgds and Hbb-b1 were up-regulated in the mcao-24h group compared with sham group and down-regulated in the mcao-48h group compared with the mcao- $24 \mathrm{~h}$ group. We also found that Rrbp1, Anxa6, Slk, Nup205 and Psmd6 changed in the mcao-48h + hUC-MSC group only compared with the mcao-48h group. Detailed protein expressions in each group are listed in Table 2. A Venn diagram is used to show the relationship of differentially expressed proteins in these groups (Fig. 1).

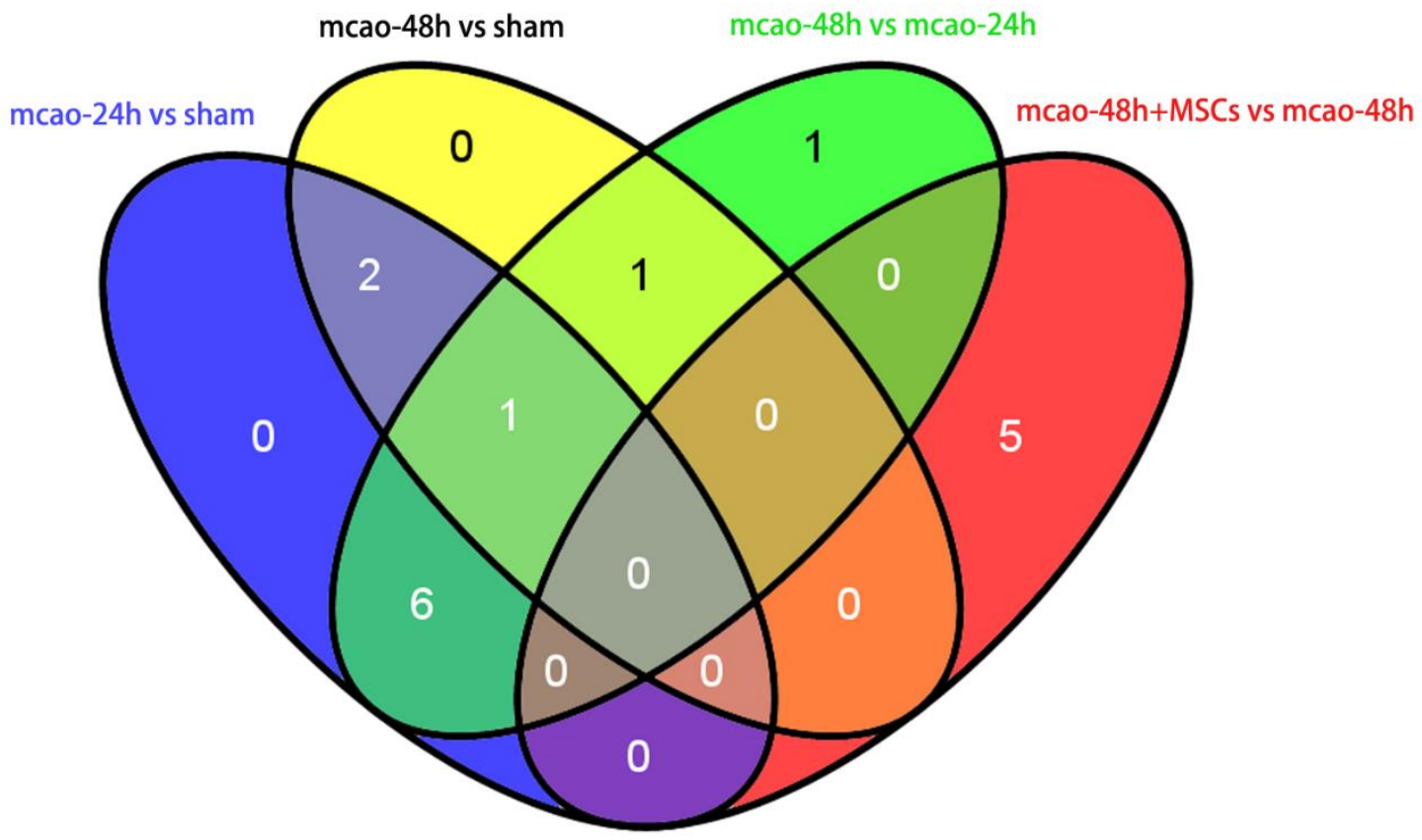

Figure 1. The number of overlapping proteins in the four groups. The blue circle represent the up-regulated and down-regulated proteins at 24 hours after cerebral ischemia compared to sham group, the yellow circle represent the up-regulated and down-regulated proteins at 48 hours after cerebral ischemia compared to sham group, the green circle represent up-regulated and down-regulated proteins at 48 hours after cerebral ischemia compared with the 24 hours after cerebral ischemia group, the red circle represent up-regulated and down-regulated proteins after hUC-MSCs treatment compared with the 48 hours after cerebral ischemia group. 

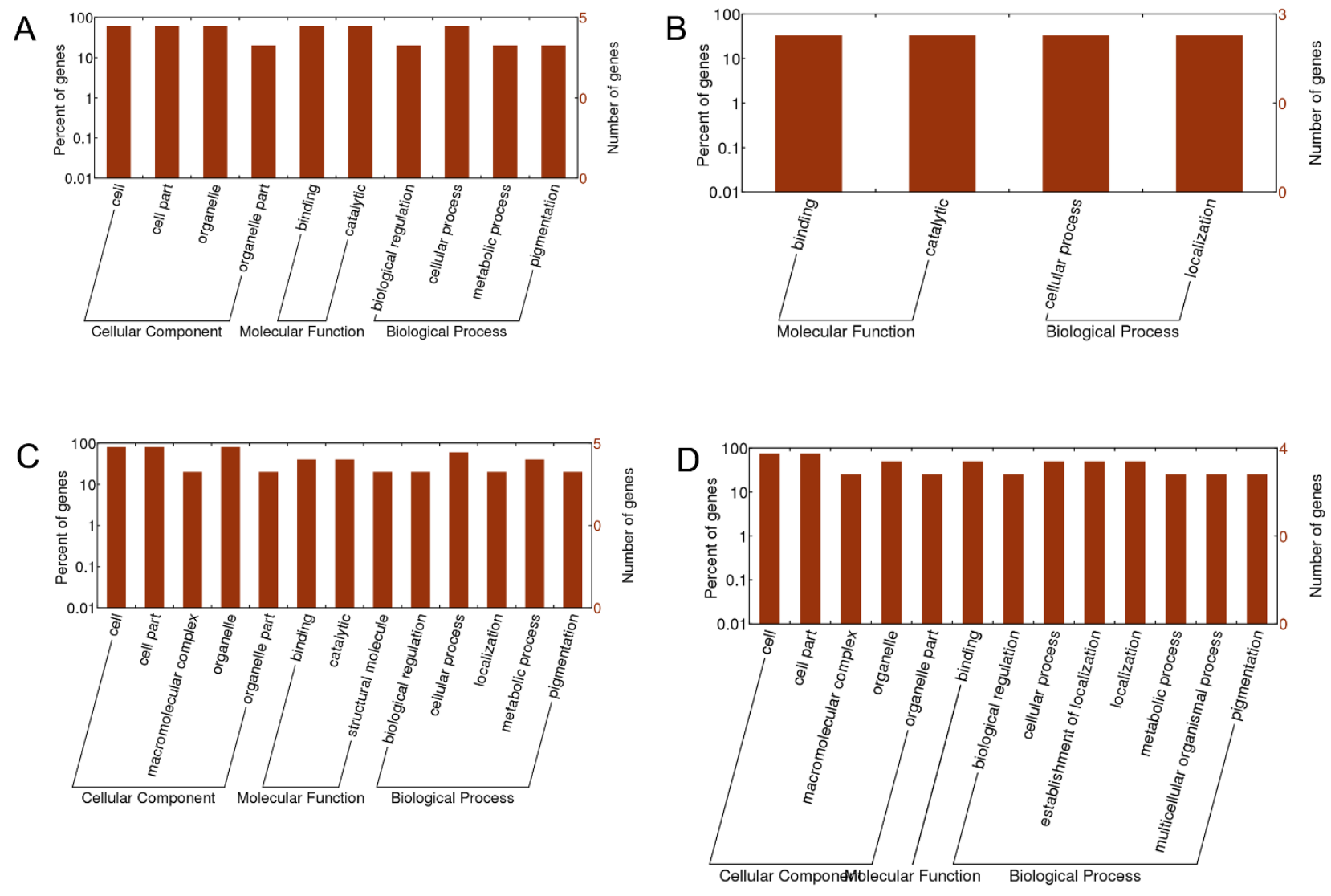

Figure 2. The GO term analysis of differentially expressed proteins in the four experimental groups. The GO term analysis of differentially expressed protein in the mcao-24h/sham group (A), the mcao-48h/sham group (B), the mcao-48h/mcao-24h group (C), and the mcao-48h + MSCs/mcao-48h group (D). Every GO term analysis shows genes involved in cellular component, molecular function and biological process.

\section{Gene Ontology analysis}

In the Gene Ontology (GO) analysis, a gene or protein could be annotated in 3 ways: cellular component, molecular function, and biological process. An analysis of GO was performed to determine the differentially expressed proteins in the four experimental groups. In the mcao-24h/sham group, the differentially expressed proteins were involved in cellular component: cell membrane, cytoplasm, nucleus and organelle; in molecular function: binding and catalytic; and in biological process: biological regulation, cellular process, metabolic process and pigmentation (Fig. 2A). In the mcao-48h/sham group, the differentially expressed proteins were involved in molecular function: binding and catalytic; and in biological process: cellular process and localization (Fig. 2B). In the mcao-48h/mcao-24h group, the differentially expressed proteins were involved in cellular component: cell membrane, cytoplasm, nucleus and organelle; in molecular function: binding, catalytic and structural molecule; and in biological process: biological regulation, cellular process, localization, metabolic process and pigmentation (Fig. 2C). In the mcao- $48 \mathrm{~h}+\mathrm{MSCs} / \mathrm{mcao}-48 \mathrm{~h}$ group, the differentially expressed proteins were involved in cellular component: cell membrane, cytoplasm, nucleus and organelle; in molecular function: binding; and in biological process: biological regulation, cellular process, establishment of localization, localization, metabolic process, multicellular organismal process and pigmentation (Fig. 2D).

\section{Network with CD200-CD200R1, CCL21-CXCR3}

The analysis of relationship between key molecules associated with neuron-glial crosstalk (CD200-CD200R1, CCL21-CXCR3) and differentially expressed proteins of these four groups are shown in Fig. 3A - 3D. 

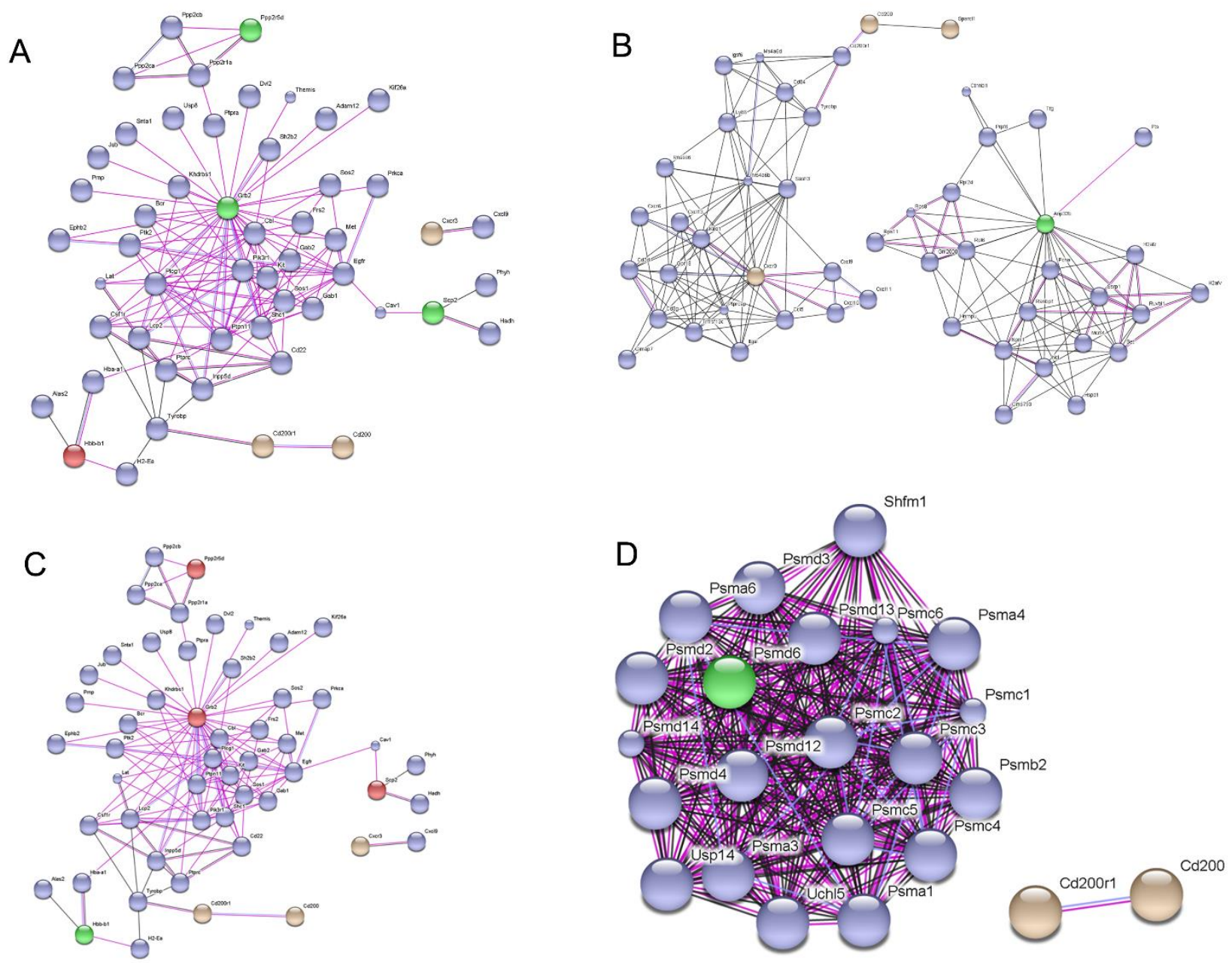

Figure 3. The network associated with CD200-CD200R1, CCL21-CXCR3 of differentially expressed proteins in the four experimental groups. The network with CD200-CD200R1 and CCL21-CXCR3 of differentially expressed proteins in the mcao$24 \mathrm{~h} / \mathrm{sham}$ group (A), the mcao-48h/sham group (B), the mcao- $48 \mathrm{~h} / \mathrm{mcao}-24 \mathrm{~h}$ group $(\mathbf{C})$, and the mcao- $48 \mathrm{~h}+\mathrm{MSCs} / \mathrm{mcao}-48 \mathrm{~h}$ group (D) are shown. Note that the trend in the mcao-24h/sham group was almost opposite to that of the mcao- $48 \mathrm{~h} / \mathrm{mcao}-24 \mathrm{~h}$ group.

\section{Network with Transcription factors}

We then analyzed the relationship between transcription factors (c-myb, Runx-1, Pu.1, Irf8, Hoxb8) and differentially expressed proteins in the four groups (Fig. 4A-4D) The transcription factors of c-myb, Runx-1, Pu.1, Irf8, and Hoxb8 are represented by myb, runx1, Spil, Irf8, and Hoxb8, respectively. We found that Grb2 is located at the center of the network.

\section{Further verification of the proteomic results}

We further confirmed the expressions of Abca13, Grb2, and Ptgds at the mRNA level using qPCR and at the protein level using ELISA.
Abca13: At the mRNA level, the Abca13 in the MCAO group was decreased at all time points compared with control group. Abca13 was at its lowest level at $6 \mathrm{~h}$ after $\mathrm{I} / \mathrm{R}$, and then reached its peak at $48 \mathrm{~h}$ after I/R and decreased after 1 week. However, after treatment with hUC-MSCs, Abca13 was increased at $24 \mathrm{~h}$ and $48 \mathrm{~h}$ after $\mathrm{I} / \mathrm{R}$ compared with the MCAO group (Fig. 5A). At the protein level, Abca13 was up-regulated in the MCAO group at all the time points compared with the control group. When treated with hUC-MSCs, Abca 13 was downregulated at $24 \mathrm{~h}$ and up-regulated at $72 \mathrm{~h}$ after $\mathrm{I} / \mathrm{R}$ compared with the MCAO group (Fig. 5B).

Grb2: At the mRNA level, Grb2 was up-regulated at $6 \mathrm{~h}$ and $12 \mathrm{~h}$, but down-regulated at $24 \mathrm{~h}, 48 \mathrm{~h}$ and $72 \mathrm{~h}$ after I/R compared with the control group. However, the 
hUC-MSC treatment could attenuate the changes at all these time points (Fig. 5C). At the protein level, Grb2 was significantly changed only at $72 \mathrm{~h}$ after I/R. The protein level of Grb2 in MCAO group was increased compared with the control group, and continually increased after hUC-MSC treatment compared with the MCAO group (Fig. 5D).

Ptgds: At the mRNA level, Ptgds was up-regulated at $6 \mathrm{~h}, 12 \mathrm{~h}$ and $48 \mathrm{~h}$, but down-regulated at $24 \mathrm{~h}$ and $72 \mathrm{~h}$ after I/R compared with control group. However, the treatment with hUC-MSCs, Ptgds was increased at $6 \mathrm{~h}, 12$ h, 24 h, 72 h, but decreased at $48 \mathrm{~h}$ after I/R (Fig. 5E). At the protein level, at $72 \mathrm{~h}$ after $\mathrm{I} / \mathrm{R}$, Ptgds expression in the MCAO group was decreased compared with the control group, which was further decreased after hUC-MSC treatment, compared with MCAO group (Fig. 5F).
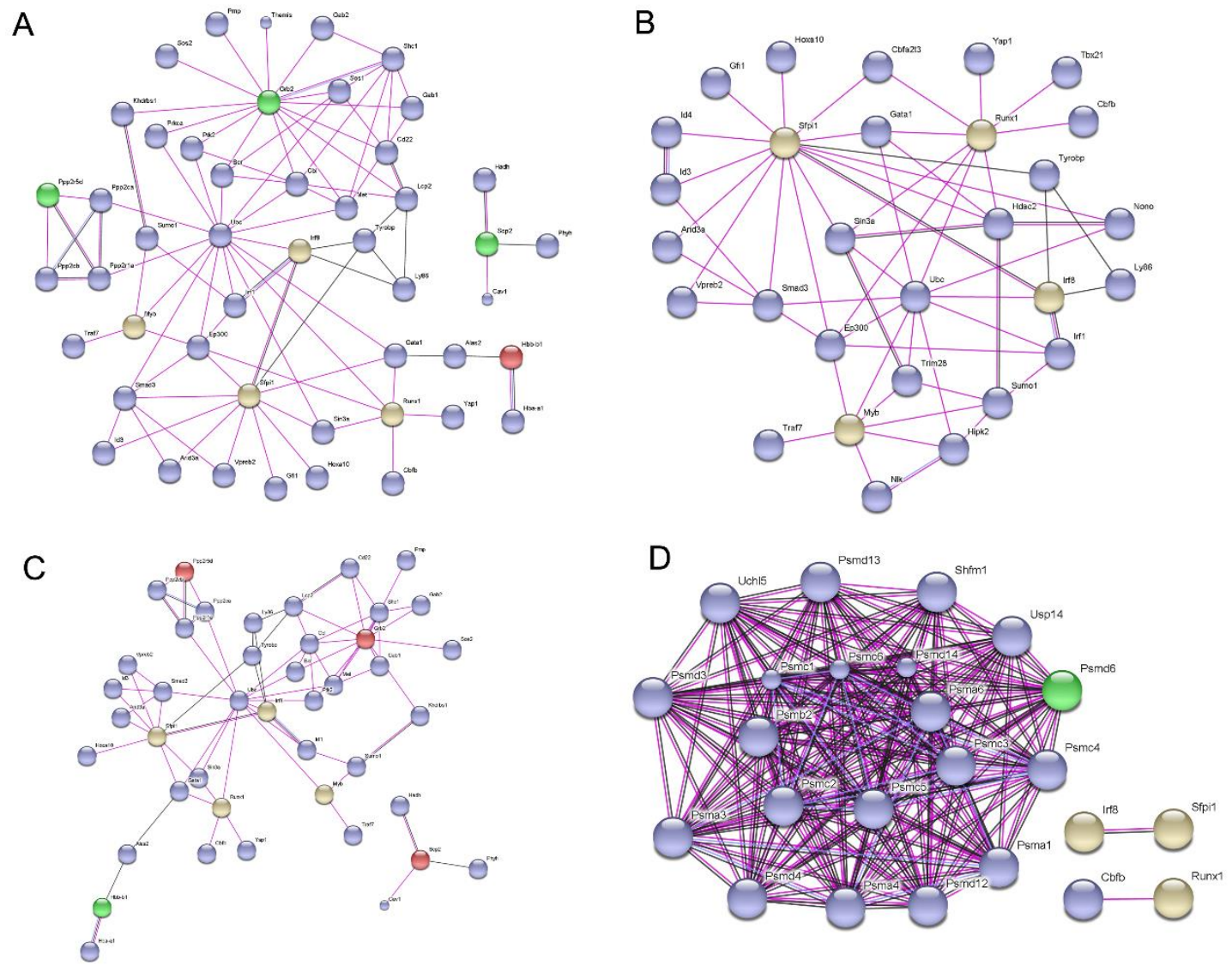

Figure 4. The network with transcription factors (c-myb, Runx-1, Pu.1, Irf8, Hoxb8) of differentially expressed proteins in the four experimental groups. The network with transcription factors (c-myb, Runx-1, Pu.1, Irf8, Hoxb8) of differential expression in the mcao-24h/sham group (A), the mcao-48h/sham group (B), the mcao- $48 \mathrm{~h} / \mathrm{mcao}-24 \mathrm{~h}$ group (C) and the mcao-48h + MSCs/mcao-48h group (D) are shown. The transcription factors of c-myb, Runx-1, Pu.1, Irf8, and Hoxb8 are represented by myb, runx1, Spi1, Irf8 and Hoxb8, respectively.

\section{DISCUSSION}

In the present study, we analyzed the differential protein expression in the ischemic brain after treatment with hUC-MSCs. A total of 16 proteins were found to be differentially expressed. The function of these proteins were further classified into two categories: 1) differentially expressed proteins during ischemic stroke and 2) differentially expressed proteins after treatment with hUC-MSCs. 


\section{Differentially expressed protein after ischemic stroke}

\section{$\underline{\text { Up-regulated proteins }}$}

Abca13 and Ptgds were up-regulated after ischemic stroke.

Abca13 is a member of the ABC gene subfamily A (ABCA). Our results showed that Abca13 was upregulated for up to $72 \mathrm{~h}$ after $\mathrm{I} / \mathrm{R}$. The expression of Abca13 is elevated in leukemia, prostate tumor, colorectal cancer, and tumor cell lines in central nervous system [16, 17]. Abca13 is thought to be a marker of poor outcome in carcinoma patients [18] as it could play a role in transport of xenobiotics and cause drug resistance. Abca 13 was also found to be associated with both schizophrenia and bipolar disorder [19]. There has been no previous report of Abca13's involvement in stroke.
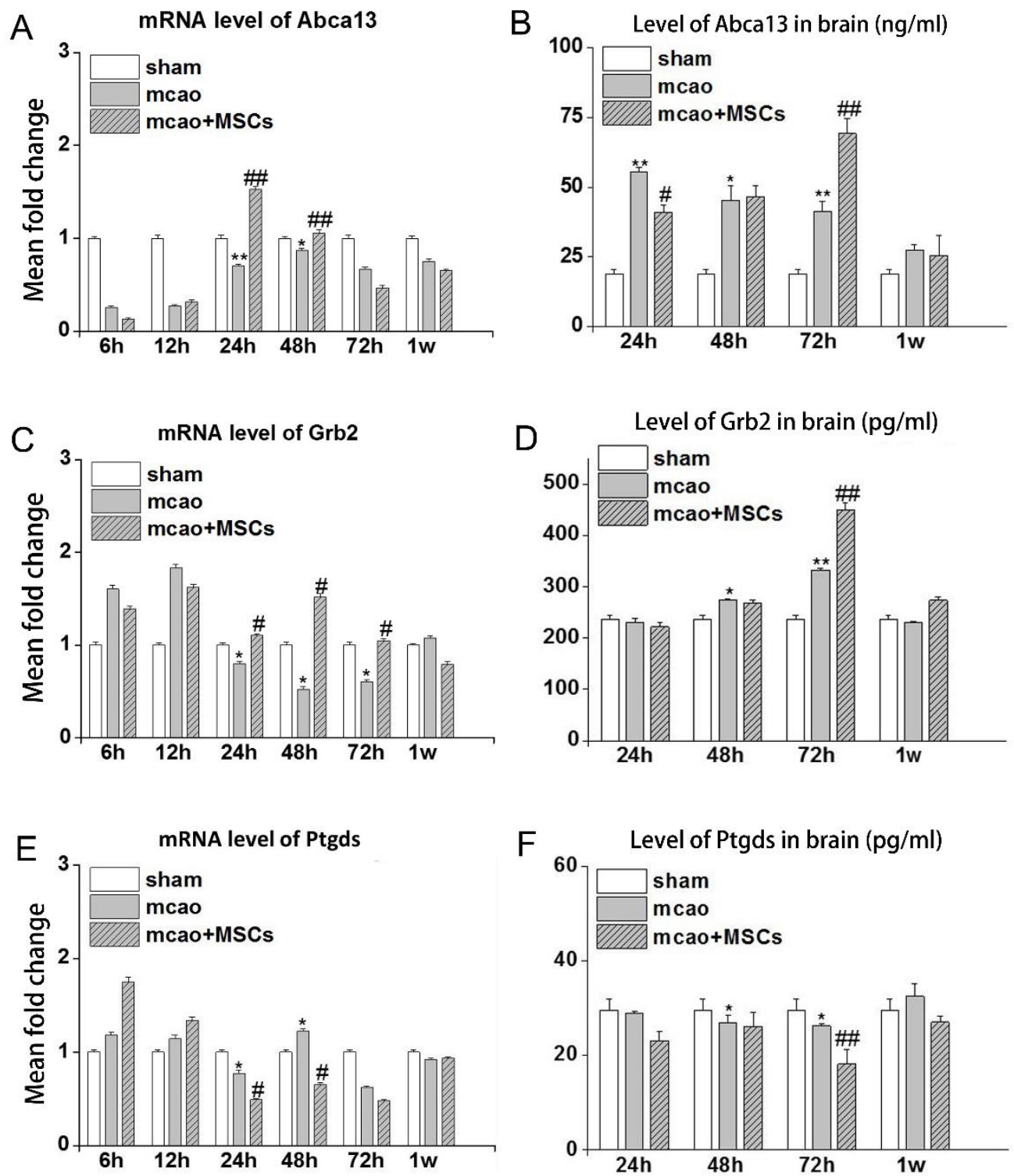

Figure 5. The mRNA and protein levels of Abca13, Grb2, Ptgds in MCAO group and MCAO + MSCs group at indicated time points. The mRNA and protein levels of Abca13 are shown in (A) and (B), respectively. The mRNA and protein levels of Grb2 are shown in (C) and (D), respectively. The mRNA and protein levels of Ptgds are shown in (E) and (F), respectively. ${ }^{*} \mathrm{p}<0.05 ;{ }^{*} \mathrm{p}<0.01$ compared with sham group; $\# \mathrm{p}<0.05 ; \# \# \mathrm{p}<0.01$ compared with MCAO group. 
Ptgds, prostaglandin D synthase, was up-regulated at $24 \mathrm{~h}$, but returned back to its normal level at $48 \mathrm{~h}$ after I/R. Ptgds could catalyze the conversion of prostaglandin $\mathrm{H} 2$ (PGH2) to prostaglandin D2 (PGD2). PGD2 is known to be involved in smooth muscle contraction/relaxation and modulate the central nervous system, inhibit platelet aggregation [20]. Ptgds is preferentially expressed in the brain, and also regulates non-rapid eye movement sleep $[21,22]$. Overexpression of Ptgds is found in patients with attention deficit hyperactivity disorder (ADHD) [23] and multiple sclerosis. One study showed Ptgds might have neuroprotective functions against neuronal death and act as an early stress protein in hypoxic-ischemic encephalopathy [24].

\section{Down-regulated proteins}

Grb2, Acot11, Acat2, Scp2, Anp32b and Ppp2r5d were down-regulated after stroke.

Grb2 mRNA was downregulated at $24 \mathrm{~h}$, and returned to the normal level at $48 \mathrm{~h}$ after I/R. However, ELISA shows that Grb2 protein expression was upregulated at 48 $\mathrm{h}$ and $72 \mathrm{~h}$ after I/R. Grb2 is a pivotal molecule in signal transduction. In network analysis, there were multiple connections between Grb2 and other proteins. Grb2 links the epidermal growth factor receptor tyrosine kinase and activate the Ras/mitogen-activated protein (MAP) kinase pathway, which subsequently activates ERK1/2 [25-27]. Grb2 also plays a role in lymphocyte development, function, and signaling [28]. Studies have shown that Grb2 overexpressed in breast cancer tissue [29, 30].

Acot11's down-regulation continued up to $48 \mathrm{~h}$ after $\mathrm{I} / \mathrm{R}$. Acat 2 and Scp2 were down-regulated at $24 \mathrm{~h}$ and returned to normal levels at $48 \mathrm{~h}$ after I/R. Acat2 and Scp2 are involved in lipid metabolism while Acot11 is involved in obesity [31-34]. Anp32b was down-regulated $48 \mathrm{~h}$ after $\mathrm{I} / \mathrm{R}$. Anp32b is a substrate for caspase-3 and plays a role in cell apoptosis [35]. There was also a report that Anp32b expression was related to worse outcome in patients with breast cancer [36]. Ppp2r5d was down-regulated $24 \mathrm{~h}$ after $\mathrm{I} / \mathrm{R}$, and returned to the normal level $48 \mathrm{~h}$ after $\mathrm{I} / \mathrm{R}$. Ppp2r5d belongs to the phosphatase 2A (PP2A) regulatory subunit $\mathrm{B}$ family, and activates PKA-mediated PP2A [37]. One study showed that its expression paralleled protein tau phosphorylation, [38], which is a major component of neurofibrillary tangles in Alzheimer's disease. Whether these proteins play a role in stroke remain uclear.

\section{Differentially expressed protein after treatment with hUC-MSCs}

$\underline{\text { Up-regulated proteins }}$
Anxa6, Slk, and Rrbp1 were up-regulated after treatment with hUC-MSCs.

Anxa6 was up-regulated significantly after treatment with hUC-MSCs. Anxa6 belongs to a family of calcium dependent protein and has a huge role in physiology and pathology. Anxa6 mediates $\mathrm{Ca}^{2+}$ flux across membranes and activate protein kinase $\mathrm{C} \alpha(\mathrm{PKC} \alpha)$ [39]. In Chlystun's study, they found that when Anxa6 expression is abolished in the cell, the mitochondria was be fragmented and respiration would be impaired [40]. hUCMSCs also inhibited apoptosis in stroke. If treatment with hUC-MSCs exerts that function through upregulating Anxa6, then the Anxa6-mediated mitochondria protection may be further explored. Depletion of Anxa6 was also found to be linked to the degradation of activated epidermal growth factor receptor (EGFR) that influences downstream signaling [41]. Anxa6 expression was also inhibited in gastric cancer cells [42, 43]. While elevated Anxa6 expression was found to increase late endosomal cholesterol levels and induce plasma membrane remodelling [44, 45]. There are reports that Anxa6 may play some role in stimulating NF- $\kappa B$, orchestrating a repair zone when the membrane is injured, and regulate terminal differentiation of chondrocytes [39, 46, 47].

Slk expression was increased significantly after treatment with hUC-MSCs. Slk plays important roles in many physiological and pathological processes. In the brain, Slk is preferentially expressed in adult neuronal cells, but not in resting astrocytes or microglia. In Zhang's study, Slk-expressing neuronal cells were decreased, while Slk was up-regulated in other cell types after MCAO [48]. These Slk expressing cells may be infiltrated leukocytes or activated native microglia. Overexpression of Slk could induce apoptosis either through a p38 or cJun N-terminal kinase-1 (JNK1)-dependent pathway [4951]. In breast cancer cells, Slk could activate HER2/Neu/ErbB2 for driving chemotaxis. Slk is also required in cell migration and cell cycle progression through G2 [52, 53]. In addition, Slk was found to be critical in embryonic development, hypertension, and tissue injury [49].

After treatment with hUC-MSCs, Rrbp1 was significantly increased. Rrbp1 is a protein in the endoplasmic reticulum (ER) membrane, which binds with ribosomes. Overexpression of Rrbp1 was found in patients with lung, colorectal, and breast cancers, of which predict an undesirable outcome [54-56]. It might inhibit apoptosis by alleviating ER stress in cancer cells. However, the role of Rrbp1in stroke is unclear.

Down-regulated proteins

Psmd6 and Nup205 were down-regulated after treatment with hUC-MSCs. 
Psmd6 is a member of the protease subunit S10 family involved in the degradation of ubiquitinated proteins and DNA repair [57]. Studies on Nup205 are limited $[58,59]$. None of them have been reported to be involved in stroke.

In this study, the mass spectrometer was used to determine the protein expression level. The information gathered from that was analyzed by software to determine differentiatal expression of proteins. We performed qPCR and ELISA to confirm proteomic results.

Abca13 is a member of $\mathrm{ABC}$ gene subfamily $\mathrm{A}$ (ABCA), which is an important member of the family in pathologic and physiologic processes in the human body. Grb2 plays an important role in signal transduction which involves in multiple pathways. Ptgds is able to catalyze the production of prostaglandin $\mathrm{D} 2$, which regulates a number of aspects in central nervous system. Because of their critical roles in pathology and physiology, we further studied these 3 proteins at the mRNA and protein levels at several time points. Our results showed that the Abca13 protein expression was consistent with the proteomic data, but not at the mRNA level. While the Grb2 expression at the protein level was inconsistent with the proteomic results, the trend of the mRNA expression pattern was similar. However, Ptgds expression at both the protein and mRNA levels were inconsistent with the proteomic data. Taken together, the data suggest that Abca13 and Grb2 may be more important in ischemic stroke after hUCMSC transplantation.

The results from the proteomic study showed 16 differentially expressed proteins. We chosed three of them to validate their expressions, and found that Abca13 and Grb2 could be of further interest.

There are some limitations regarding the present research. Protein validation was not conducted for each of the differentially expressed proteins. Hence, proteins that may be of interest for the treatment of stroke were not identified yet. Proteomic results for Abca13, Grb2 and Ptgds showed that Abca13 at the protein level and Grb2 at the mRNA level were consistent with the proteomic study; their functions will be determined in the future.

\section{Conclusion}

In the present study, we analyzed the expression profiles of proteins after stroke in the presence or absence of hUCMSCs treatment using iTRAQ. We identified 16 differentially expressed proteins. There were complicated connections between them and transcription factors or other proteins. Proteomic validation results showed that Abca13 and Grb2 are of interest and could be further pursued. Many of these proteins that are highlighted in this study have not been reported in the field of stroke before. We believe the proteomic method used in the present study could provide clues for the involvement of these proteins in stroke, which could help us further understand stroke and facilitate treatment with hUCMSCs for ischemic stroke patients.

\section{Acknowledgement}

This work was supported by the National Natural Science Foundation of China (81100863, 81230026, 81171085), the Natural Science Foundation (BL2012013) and the Bureau of Health (LJ201101) of Jiangsu Province of China.

\section{References}

[1] Bang OY, Lee JS, Lee PH, Lee G (2005). Autologous mesenchymal stem cell transplantation in stroke patients. Ann Neurol, 57: 874-882

[2] Kim SS, Yoo SW, Park TS, Ahn SC, Jeong HS, Kim JW, et al. (2008). Neural induction with neurogenin1 increases the therapeutic effects of mesenchymal stem cells in the ischemic brain. Stem Cells, 26: 2217-2228

[3] Koh SH, Kim KS, Choi MR, Jung KH, Park KS, Chai YG, et al. (2008). Implantation of human umbilical cordderived mesenchymal stem cells as a neuroprotective therapy for ischemic stroke in rats. Brain Res, 1229: 233248

[4] Lee JS, Hong JM, Moon GJ, Lee PH, Ahn YH, Bang OY (2010). A long-term follow-up study of intravenous autologous mesenchymal stem cell transplantation in patients with ischemic stroke. Stem Cells, 28: 1099-1106

[5] Liu H, Honmou O, Harada K, Nakamura K, Houkin K, Hamada H, et al. (2006). Neuroprotection by PIGF genemodified human mesenchymal stem cells after cerebral ischaemia. Brain, 129: 2734-2745

[6] Quittet MS, Touzani O, Sindji L, Cayon J, Fillesoye F, Toutain J, et al. (2015). Effects of mesenchymal stem cell therapy, in association with pharmacologically active microcarriers releasing VEGF, in an ischaemic stroke model in the rat. Acta Biomater, 15: 77-88

[7] Yoo SW, Chang DY, Lee HS, Kim GH, Park JS, Ryu $\mathrm{BY}$, et al. (2013). Immune following suppression mesenchymal stem cell transplantation in the ischemic brain is mediated by TGF-beta. Neurobiol Dis, 58: 249257

[8] Borlongan CV, Glover LE, Tajiri N, Kaneko Y, Freeman TB (2011). The great migration of bone marrow-derived stem cells toward the ischemic brain: therapeutic implications for stroke and other neurological disorders. Prog Neurobiol, 95: 213-228

[9] Bronckaers A, Hilkens P, Martens W, Gervois P, Ratajczak J, Struys T, et al. (2014). Mesenchymal stem/stromal cells as a pharmacological and therapeutic approach to accelerate angiogenesis. Pharmacol Ther, 143: $181-196$

[10] Honmou O, Onodera R, Sasaki M, Waxman SG, Kocsis JD (2012). Mesenchymal stem cells: therapeutic outlook for stroke. Trends Mol Med, 18: 292-297 
[11] Li Y, Hu G, Cheng Q (2015). Implantation of human umbilical cord mesenchymal stem cells for ischemic stroke: perspectives and challenges. Front Med, 9: 20-29

[12] Cheng Q, Zhang Z, Zhang S, Yang H, Zhang X, Pan J, et al. (2015). Human umbilical cord mesenchymal stem cells protect against ischemic brain injury in mouse by regulating peripheral immunoinflammation. Brain Res, 1594: 293-304

[13] Xu Y, Zhang W, Klaus J, Young J, Koerner I, Sheldahl LC, et al. (2006). Role of cocaine- and amphetamineregulated transcript in estradiol-mediated neuroprotection. Proc Natl Acad Sci U S A, 103: 1448914494

[14] Liu R, Zhang Z, Lu Z, Borlongan C, Pan J, Chen J, et al. (2013). Human umbilical cord stem cells ameliorate experimental autoimmune encephalomyelitis by regulating immunoinflammation and remyelination. Stem Cells Dev, 22: 1053-1062

[15] Ji YH, Ji JL, Sun FY, Zeng YY, He XH, Zhao JX, et al. (2010). Quantitative Proteomics Analysis of Chondrogenic Differentiation of C3H10T1/2 Mesenchymal Stem Cells by iTRAQ Labeling Coupled with On-line Two-dimensional LC/MS/MS. Mol Cell Proteomics., 9: 550-564

[16] Albrecht C, Viturro E (2007). The ABCA subfamily-gene and protein structures, functions and associated hereditary diseases. Pflugers Arch, 453: 581-589

[17] Hlavata I, Mohelnikova-Duchonova B, Vaclavikova R, Liska V, Pitule P, Novak P, et al. (2012). The role of $\mathrm{ABC}$ transporters in progression and clinical outcome of colorectal cancer. Mutagenesis, 27: 187-196

[18] Nymoen DA, Holth A, Hetland Falkenthal TE, Trope CG, Davidson B (2015). CIAPIN1 and ABCA13 are markers of poor survival in metastatic ovarian serous carcinoma. Mol Cancer, 14: 44

[19] Knight HM, Pickard BS, Maclean A, Malloy MP, Soares DC, McRae AF, et al. (2009). A cytogenetic abnormality and rare coding variants identify ABCA13 as a candidate gene in schizophrenia, bipolar disorder, and depression. Am J Hum Genet, 85: 833-846

[20] Lim W, Bae SM, Jo G, Bazer FW, Choi Y, Song G (2015). Prostaglandin D(2) synthase related to estrogen in the female reproductive tract. Biochem Biophys Res Commun, 456: 355-360

[21] Eguchi N, Kuwahata Y, Mochizuki T, Urade Y, Hayaishi $\mathrm{O}$ (2001). Prostaglandin D synthase gene is involved in the regulation of non-REM sleep. Society for Neuroscience Abstracts, 27: 1380-1380

[22] Pinzar E, Kanaoka Y, Inui T, Eguchi N, Urade Y, Hayaishi O (2000). Prostaglandin D synthase gene is involved in the regulation of non-rapid eye movement sleep. Proc Natl Acad Sci U S A, 97: 4903-4907

[23] Marin-Mendez JJ, Patino-Garcia A, Segura V, Ortuno F, Galvez MD, Soutullo CA (2012). Differential expression of prostaglandin D2 synthase (PTGDS) in patients with attention deficit-hyperactivity disorder and bipolar disorder. J Affect Disord, 138: 479-484

[24] Taniguchi H, Mohri I, Okabe-Arahori H, Kanekiyo T, Kagitani-Shimono K, Wada K, et al. (2007). Early induction of neuronal lipocalin-type prostaglandin D synthase after hypoxic-ischemic injury in developing brains. Neurosci Lett, 420: 39-44

[25] Ahmed Z, Timsah Z, Suen KM, Cook NP, Lee GRt, Lin $\mathrm{CC}$, et al. (2015). Grb2 monomer-dimer equilibrium determines normal versus oncogenic function. Nat Commun, 6: 7354

[26] Gale NW, Kaplan S, Lowenstein EJ, Schlessinger J, BarSagi D (1993). Grb2 mediates the EGF-dependent activation of guanine nucleotide exchange on Ras. Nature, 363: 88-92

[27] Tari AM, Lopez-Berestein G (2001). GRB2: a pivotal protein in signal transduction. Semin Oncol, 28: 142-147

[28] Jang IK, Zhang J, Gu H (2009). Grb2, a simple adapter with complex roles in lymphocyte development, function, and signaling. Immunol Rev, 232: 150-159

[29] Daly RJ, Binder MD, Sutherland RL (1994). Overexpression of the Grb2 gene in human breast cancer cell lines. Oncogene, 9: 2723-2727

[30] Yip SS, Crew AJ, Gee JM, Hui R, Blamey RW, Robertson JF, et al. (2000). Up-regulation of the protein tyrosine phosphatase SHP-1 in human breast cancer and correlation with GRB2 expression. Int J Cancer, 88: 363368

[31] Huang H, Ball JM, Billheimer JT, Schroeder F (1999). The sterol carrier protein-2 amino terminus: a membrane interaction domain. Biochemistry, 38: 13231-13243

[32] Pedrelli M, Davoodpour P, Degirolamo C, Gomaraschi M, Graham M, Ossoli A, et al. (2014). Hepatic ACAT2 knock down increases ABCA1 and modifies HDL metabolism in mice. PLoS One, 9: e93552

[33] Zhang J, Kelley KL, Marshall SM, Davis MA, Wilson MD, Sawyer JK, et al. (2012). Tissue-specific knockouts of ACAT2 reveal that intestinal depletion is sufficient to prevent diet-induced cholesterol accumulation in the liver and blood. J Lipid Res, 53: 1144-1152

[34] Zhang Y, Li Y, Niepel MW, Kawano Y, Han S, Liu S, et al. (2012). Targeted deletion of thioesterase superfamily member 1 promotes energy expenditure and protects against obesity and insulin resistance. Proc Natl Acad Sci U S A, 109: 5417-5422

[35] Yu Y, Shen SM, Zhang FF, Wu ZX, Han B, Wang LS (2012). Acidic leucine-rich nuclear phosphoprotein 32 family member B (ANP32B) contributes to retinoic acidinduced differentiation of leukemic cells. Biochem Biophys Res Commun, 423: 721-725

[36] Reilly PT, Afzal S, Gorrini C, Lui K, Bukhman YV, Wakeham A, et al. (2011). Acidic nuclear phosphoprotein $32 \mathrm{kDa}$ (ANP32)B-deficient mouse reveals a hierarchy of ANP32 importance in mammalian development. Proc Natl Acad Sci U S A, 108: 1024310248

[37] Yu UY, Ahn JH (2010). Phosphorylation on the PPP2R5D B regulatory subunit modulates the biochemical properties of protein phosphatase $2 \mathrm{~A}$. BMB Rep, 43: 263-267

[38] Yu UY, Yoo BC, Ahn JH (2014). Regulatory B Subunits of Protein Phosphatase 2A Are Involved in Site-specific Regulation of Tau Protein Phosphorylation. Korean J Physiol Pharmacol, 18: 155-161

[39] Minashima T, Small W, Moss SE, Kirsch T (2012). 
Intracellular modulation of signaling pathways by annexin A6 regulates terminal differentiation of chondrocytes. J Biol Chem, 287: 14803-14815

[40] Chlystun M, Campanella M, Law AL, Duchen MR, Fatimathas L, Levine TP, et al. (2013). Regulation of mitochondrial morphogenesis by annexin A6. PLoS One, 8: e53774

[41] Koumangoye RB, Nangami GN, Thompson PD, Agboto VK, Ochieng J, Sakwe AM (2013). Reduced annexin A6 expression promotes the degradation of activated epidermal growth factor receptor and sensitizes invasive breast cancer cells to EGFR-targeted tyrosine kinase inhibitors. Mol Cancer, 12: 167

[42] Qi Y, Zhang X, Kang Y, Wu J, Chen J, Li H, et al. (2015). Genome-wide transcriptional profiling analysis reveals annexin A6 as a novel EZH2 target gene involving gastric cellular proliferation. Mol Biosyst, 11: 1980-1986

[43] Wang X, Zhang S, Zhang J, Lam E, Liu X, Sun J, et al. (2013). Annexin A6 is down-regulated through promoter methylation in gastric cancer. Am J Transl Res, 5: 555562

[44] Alvarez-Guaita A, Vila de Muga S, Owen DM, Williamson D, Magenau A, Garcia-Melero A, et al. (2015). Evidence for annexin A6-dependent plasma membrane remodelling of lipid domains. $\mathrm{Br} \quad \mathrm{J}$ Pharmacol, 172: 1677-1690

[45] Musiol A, Gran S, Ehrhardt C, Ludwig S, Grewal T, Gerke V, et al. (2013). Annexin A6-balanced late endosomal cholesterol controls influenza A replication and propagation. MBio, 4: e00608-00613

[46] Campbell KA, Minashima T, Zhang Y, Hadley S, Lee YJ, Giovinazzo J, et al. (2013). Annexin A6 interacts with p65 and stimulates NF-kappaB activity and catabolic events in articular chondrocytes. Arthritis Rheum, 65: 3120-3129

[47] Swaggart KA, Demonbreun AR, Vo AH, Swanson KE, Kim EY, Fahrenbach JP, et al. (2014). Annexin A6 modifies muscular dystrophy by mediating sarcolemmal repair. Proc Natl Acad Sci U S A, 111: 6004-6009

[48] Zhang YH, Hume K, Cadonic R, Thompson C, Hakim A, Staines W, et al. (2002). Expression of the Ste20-like kinase SLK during embryonic development and in the murine adult central nervous system. Brain Res Dev Brain Res, 139: 205-215

[49] Al-Zahrani KN, Baron KD, Sabourin LA (2013). Ste20like kinase SLK, at the crossroads: a matter of life and death. Cell Adh Migr, 7: 1-10

[50] Hao W, Takano T, Guillemette J, Papillon J, Ren G, Cybulsky AV (2006). Induction of apoptosis by the Ste20-like kinase SLK, a germinal center kinase that activates apoptosis signal-regulating kinase and p38. J Biol Chem, 281: 3075-3084

[51] Sabourin LA, Tamai K, Seale P, Wagner J, Rudnicki MA (2000). Caspase 3 cleavage of the Ste20-related kinase SLK releases and activates an apoptosis-inducing kinase domain and an actin-disassembling region. Mol Cell Biol, 20: 684-696

[52] O'Reilly PG, Wagner S, Franks DJ, Cailliau K, Browaeys E, Dissous C, et al. (2005). The Ste20-like kinase SLK is required for cell cycle progression through G2. J Biol Chem, 280: 42383-42390

[53] Quizi JL, Baron K, Al-Zahrani KN, O'Reilly P, Sriram RK, Conway J, et al. (2013). SLK-mediated phosphorylation of paxillin is required for focal adhesion turnover and cell migration. Oncogene, 32: 4656-4663

[54] Liang X, Sun S, Zhang X, Wu H, Tao W, Liu T, et al. (2015). Expression of ribosome-binding protein 1 correlates with shorter survival in Her-2 positive breast cancer. Cancer Sci, 106: 740-746

[55] Pan Y, Cao F, Guo A, Chang W, Chen X, Ma W, et al. (2015). Endoplasmic reticulum ribosome-binding protein 1, RRBP1, promotes progression of colorectal cancer and predicts an unfavourable prognosis. $\mathrm{Br} \mathrm{J}$ Cancer,

[56] Tsai HY, Yang YF, Wu AT, Yang CJ, Liu YP, Jan YH, et al. (2013). Endoplasmic reticulum ribosome-binding protein 1 (RRBP1) overexpression is frequently found in lung cancer patients and alleviates intracellular stressinduced apoptosis through the enhancement of GRP78. Oncogene, 32: 4921-4931

[57] Narayanaswamy PB, Hodjat M, Haller H, Dumler I, Kiyan Y (2014). Loss of urokinase receptor sensitizes cells to DNA damage and delays DNA repair. PLoS One, 9: e101529

[58] Galy V, Mattaj IW, Askjaer P (2003). Caenorhabditis elegans nucleoporins Nup93 and Nup205 determine the limit of nuclear pore complex size exclusion in vivo. Mol Biol Cell, 14: 5104-5115

[59] Lu Y, Kucharski TJ, Gamache I, Blanchette P, Branton PE, Teodoro JG (2014). Interaction of adenovirus type 5 E4orf4 with the nuclear pore subunit Nup205 is required for proper viral gene expression. J Virol, 88: 1324913259 